\title{
RELACIÓN ENTRE CLOROFILA-A Y LAS VARIABLES OCEANOGRÁFICAS EN EL ÁREA PERIGLACIAR DEL SENO GALLEGOS (CORDILLERA DARWIN, CHILE): BAJO CONDICIONES INVERNALES
}

\author{
THE RELATIONSHIPS BETWEEN CHLOROPHYLL-A AND OCEANOGRAPHIC \\ VARIABLES IN THE PERIGLACIAL AREA OF THE SENO GALLEGOS \\ (CORDILLERA DARWIN, CHILE): UNDER WINTER CONDITIONS
}

Osvaldo Vásquez ${ }^{1}$, Santiago Pineda ${ }^{1}$, Eduardo Quiroga ${ }^{2}$, Bibiana Jara $^{3}$ \& Américo Montiel ${ }^{1,4}$

\begin{abstract}
The relationships between chlorophyll a concentration, physical-chemical variables (temperature, salinity, density, total suspended particulate matter) and nutrients (silicate, nitrate, nitrite and phosphate)

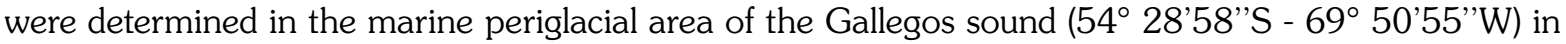
August 2010 (austral winter). Twelve stations were studied out directly from the glacier edge to mouth of the sound. At each station, a CTD was deployed and water samples were collected. The water temperatures ranged from 3.74 to $6.55^{\circ} \mathrm{C}$, the salinity from 28.39 to 30.14 psu and chlorophyll a concentrations varied from 0.24 to $2.69 \mathrm{mg} \mathrm{m}^{-3}$; the total particulate matter concentrations varied between 0.12 and $14.0 \mathrm{mg} \mathrm{L}^{-1}$. The silicate concentrations varied between 2.58 and $9.57 \mu \mathrm{M}$, phosphate between 0.01 and $8.02 \mu \mathrm{M}$, and nitrate and nitrite values ranged from 0.02 to $30.34 \mu \mathrm{M}$ and 0.01 to $2.99 \mu \mathrm{M}$, respectively.

The chlorophyll a concentration in the sound was significantly correlated with depth, total particulate matter and density $(\rho=0.477)$, whereas lower correlation values resulted with silicate and phosphate $(\rho$ $=0.463)$. The lowest significant correlation value was observed with nitrate concentrations $(\rho=0.397)$. Our result suggest that the water column stratification is linked to total particular matter maintaining high chlorophyll a concentrations in the euphotic layer of this cold water estuarine system.

Key words: Brookes Bay, Patagonia, oceanography, particulate matter, estuarine.

1 Laboratorio de Ecología y Ciencias Ambientales, Instituto de la Patagonia, Universidad de Magallanes, Punta Arenas, Chile. ovasquez@umag.cl.

2 Pontificia Universidad Católica de Valparaíso, Escuela de Ciencias del Mar, Casilla 1020, Av. Altamirano 1480, Valparaíso, Chile.

3 Facultad de Ciencias, Universidad de Magallanes, Punta Arenas, Chile.

4 Fundación Centro de Estudios del Cuaternario de Fuego - Patagonia y Antártica, Punta Arenas, Chile.
\end{abstract}




\section{RESUMEN}

Se determinó la relación entre la concentración de clorofila a, las variables físico-químicas (temperatura, salinidad, densidad y solidos totales suspendidos) y nutrientes (silicatos, nitratos, nitritos y fosfatos) en el área periglaciar de seno Gallegos (5428'58'S - 69 50'55” W) en agosto del 2010 (invierno austral). Doce estaciones fueron estudiadas directamente desde el borde glaciar a la boca del seno Gallegos. En cada una de las estaciones se realizaron lances de CTD y se colectaron muestras de agua. La columna de agua en seno Gallegos presentó un rango de temperatura de $3,74{ }^{\circ} \mathrm{C}$ a $6,55{ }^{\circ} \mathrm{C}$, el rango de salinidad fue de 28,39 a 30,14 psu y las concentraciones de clorofila a variaron desde 0,24 hasta 2,69 $\mathrm{mg} \mathrm{m}^{-3}$; la concentración de solidos totales suspendidos varió entre 0,12 y $14,0 \mathrm{mg} \mathrm{L}^{-1}$. Las concentraciones de silicato variaron entre 2,58 hasta $9,57 \mu \mathrm{M}$, los fosfatos variaron de 0,01 a 8,02 $\mu \mathrm{M}$, mientras que los valores de nitratos y nitritos variaron de 0,02 a 30,34 $\mu \mathrm{M}$ y 0,01 a 2,99 $\mu \mathrm{M}$, respectivamente. Las concentraciones de clorofila $a$ en el seno se correlacionaron significativamente con la profundidad, solidos totales suspendidos y la densidad $(\rho=0,477)$, mientras que valores bajos de correlación resultaron con los silicatos y fosfatos $(\rho=0,463)$. Los valores con menor correlación significativa fueron observados en los nitratos $(\rho=0,397)$. Nuestros resultados sugieren que la estratificación de la columna de agua se vincula con los solidos totales suspendidos manteniendo altas las concentraciones de clorofila a en la capa eufótica de estos sistemas estuarinos fríos.

Palabras clave: bahía Brookes, Patagonia, oceanografía, sólidos suspendidos, estuario.

\section{INTRODUCCIÓN}

Con un área de $240.000 \mathrm{~km}^{2}$, el sistema de fiordos y canales chilenos ( $41^{\circ} 30^{\prime} \mathrm{S}$ - $56^{\circ} \mathrm{S}$; Silva et al. 2001) es considerado el ecosistema estuarino templado frio más extenso en el hemisferio sur (Antezana 1999; Sobarzo 2009). Esta región es caracterizada por la presencia de grandes campos de hielo (e.g., Campo de Hielo Patagónico Norte y Sur, Gran Campo Nevado y Campo de Hielo de la Cordillera Darwin) que contribuyen significativamente con agua dulce y nutrientes a los ecosistemas costeros de la región, regulando los niveles de producción primaria (González et al. 2011; Torres et al. 2011). En la actualidad, estos sistemas hidrológicos tienen un alto interés para el desarrollo productivo de la región, asociado principalmente al potencial hidroeléctrico, turismo y acuicultura (Pantoja et al. 2011). Además, en las últimas décadas las características oceanográficas en la región están siendo modificadas por el aumento de la escorrentía de agua dulce inducidos por el incremento de la temperatura global (Rignot et al. 2003). Estas características han impulsado un mayor interés en estudiar los procesos oceanográficos en la región con el fin de comprender los posibles cambios ecológicos, a futuro, en uno de los ecosistemas marinos más prístinos del mundo (Montiel \& Ríos 2010).
En los fiordos y canales chilenos, se ha determinado que la concentración de Clorofila a (Chl-a) en la columna de agua depende tanto de la capacidad de penetración de la luz (Ramírez 2005) como de la disponibilidad de nutrientes en la columna de agua (Torres et al. 2011). A su vez, la penetración de la luz es influenciada por la presencia de los sólidos suspendidos en la columna de agua, provenientes de distintas fuentes terrígenas (Castro \& Huber 2003; McLusky \& Elliot 2004; Iriarte et al. 2007; Kilian et al. 2007; González et al. 2011; Torres et al. 2011).

Por otra parte, estudios sobre el efecto de la turbidez y nutrientes sobre los patrones de distribución espaciales y temporal del fitoplancton (i.e., Chl-a) en una área periglaciar son muy escasos y son de alto interés científico, dado que permiten entender como los procesos de mezcla y estratificación de la columna de agua, y la intensidad de la luz afectan sobre la dinámica de la producción primaria en estos sistemas estuarinos fríos.

En términos oceanográficos, la zona sur de los fiordos y canales chilenos (sensu Silva et al. 2001), se encuentra influenciada constantemente por el ingreso de aguas oceánicas de origen subantártico, las que se mezclan con aguas interiores más frías y menos salinas provenientes de lluvias, ríos y deshielos cordilleranos de glaciares (Guzmán \& Silva 2002; Sievers et al. 2002, 
Sievers 2006). El agua que ingresa, se mezcla bajo la capa superficial con una capa de agua dulce, induciendo una alta estratificación vertical en la columna de agua, lo que determina las características físicas y biológicas de los sistemas de fiordos (Calvete \& Sobarzo 2011). Históricamente, los estudios oceanográficos se han realizado con el objetivo de describir las características hidrológicas e incrementar el conocimiento de la fauna marina en el ecosistema de fiordos y canales chilenos (Murray 1895; Pickard 1971; Chuecas \& Ahumada 1980; Faranda \& Guglielmo 1991; Rojas 1993 fide Silva \& Palma 2006; Antezana 1999; Saggiomo et al. 2011). Estas expediciones han sido realizadas mayoritariamente durante la temporada primaveraverano y en áreas relativamente profundas ( $>50$ m) (Vásquez 2011²). Por lo que, el comportamiento de los distintos procesos oceanográficos durante la época invernal en áreas adyacentes a glaciares son prácticamente desconocidas.

En este contexto, el presente estudio investigó cuales son las variables oceanográficas que están influenciando la concentración y distribución de Chl-a frente a un glaciar bajo condiciones invernales. Los objetivos del presente estudio fueron: (1) caracterizar las variables físico-químicas de la columna de agua en el sector de seno Gallegos en términos de la

1 Faranda, F. \& L. Guglielmo. 1991. Oceanographic cruise February-March 1991, Strait of Magellan. R/V "Cariboo", Data Report-Part I. Physical, chemical and biological oceanography, 192.

2 Tesis de pregrado carrera Biología Marina de título "Evaluación espacial y temporal de la influencia de un glaciar sobre características oceanográficas en Seno Gallegos (Seno Almirantazgo, Chile)". temperatura, salinidad y densidad, (2) describir los patrones de distribución de las concentraciones de Chl- $a$, sólidos totales suspendidos (STS) y nutrientes y (3) determinar la relación entre la distribución espacial de la concentración de Chl-a, los STS y los nutrientes (silicatos, fosfatos, nitritos, nitratos).

\section{MATERIAL Y MÉTODOS}

Área de estudio

El seno Gallegos (54²8'58'S - 6950'51,88'W; Fig. 1) se ubica en un sistema de cuencas aledaño a bahía Brookes (Fig. 1). En el extremo nororiental del seno Gallegos se encuentra el ventisquero Garibaldi proveniente del Campo de Hielo de la Cordillera Darwin. Por lo que se espera que este glaciar sea la fuente principal de descarga de hielo y escorrentía de agua dulce hacia el área periglaciar marina. Además, se observó que durante el muestreo, la cuenca del seno Gallegos se presentó parcialmente cubierta por una capa de hielo, la cual cubría en su totalidad la primera porción del seno hasta un kilómetro de longitud. Mientras que el resto del seno presentó una delgada capa de escarcha superficial.

Obtención de datos hidrográficos y muestras de agua

El muestreo se realizó a bordo de la L/M Cabo Tamar entre el 16 y 18 de agosto del 2010 en condiciones diurnas del invierno austral. En el área de estudio, se establecieron 12 estaciones

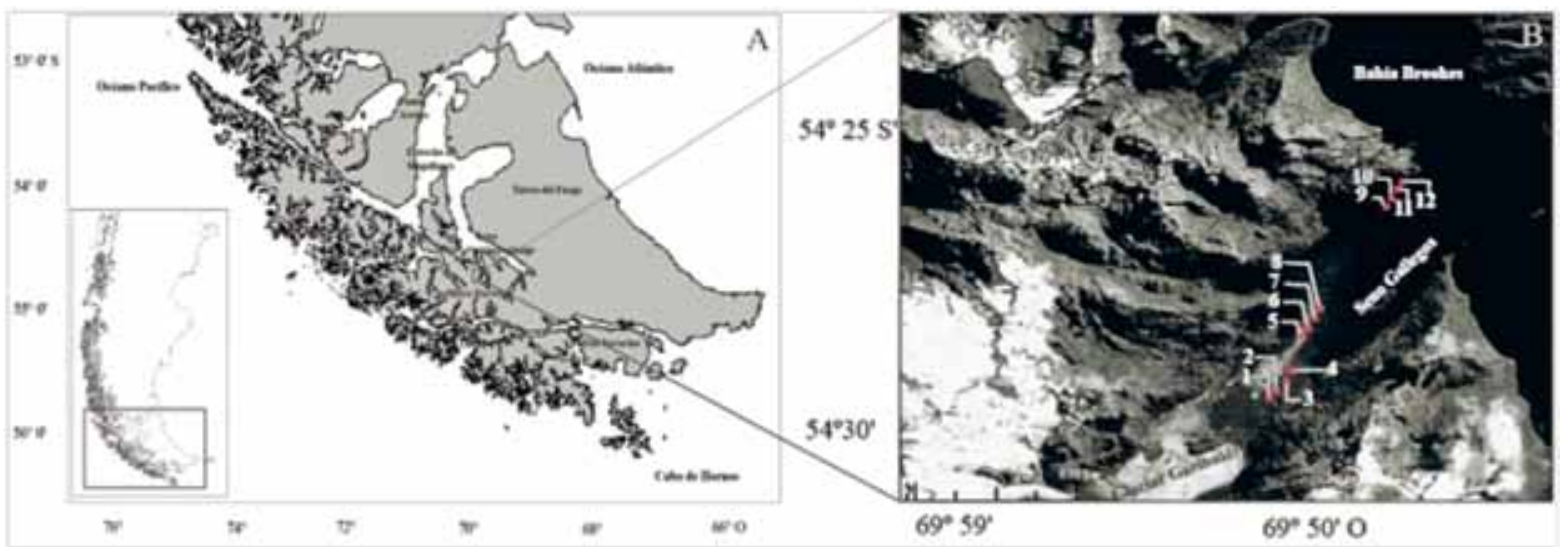

Fig. 1. Ubicación geográfica de los lances de CTD y botella Niskin realizados entre el 16 y 18 de agosto del 2010 en condiciones diurnas del invierno austral en el seno Gallegos (Cordillera Darwin, Chile). 
de muestreo (E1 a E12; Tabla 1) perpendiculares al glaciar (Fig. 1). El registro de temperatura $\left({ }^{\circ} \mathrm{C}\right.$ ), salinidad (psu), densidad (sigma-t; $\mathrm{kg} \mathrm{m}^{-3}$ ) y Chl-a $\left(\mathrm{mg} \mathrm{m}^{-3}\right)$ se obtuvieron mediante un CTD Seabird (SBE19 plus), equipado con sensores de fluorescencia (Wet labs Wetstar).

La recolección de muestras de agua para la determinación de los STS y nutrientes (silicato, nitrato, nitrito y fosfato) se realizó utilizando una botella Niskin ( $5 \mathrm{~L}$ de capacidad). En cada estación, se recolectaron muestras de agua a profundidades discretas de de 0, 5, 10, 20 y 30 m de profundidad. Los lances con botella Niskin se realizaron en la misma ubicación de los lances del CTD. Todas las muestras de agua recolectadas se almacenaron en envases de polietileno para su posterior análisis.

Análisis laboratorio

El contenido de STS se determinó mediante el método gravimétrico. Para esto, en el laboratorio se filtraron 0,4 L de muestra de agua a través de un filtro de policarbonato con un tamaño de poro de $0,45 \mu \mathrm{m}$, previamente pesado. Posteriormente, los filtros se secaron en una estufa a $60^{\circ} \mathrm{C}$ por 24 hrs. El incremento en el peso del filtro representa los STSexpresados en $\mathrm{mg} \mathrm{L}^{-1}$ (Eaton et al. 2005), de acuerdo a la fórmula (1)

$$
\text { (1)STS }(\mathrm{mg})=\frac{(A-B) \times 100}{\text { Vol.Muestra }(L)}
$$

\section{Donde:}

A: peso del filtro+ residuo seco $(\mathrm{mg})$

B: peso del filtro (mg)

Las muestras de nutrientes fueron filtradas a bordo de la embarcación a través de un filtro de policarbonato con un tamaño de poro de $0,45 \mu \mathrm{m} y$ analizadas mediante el método colorimétrico según Strickland \& Parsons (1968) para silicatos, mediante método espectrofotométrico de acuerdo a la metodología por Strickland \& Parsons (1972) para fosfatos y por Parsons et al. (1984) para nitrito y nitrato.

Análisis de datos

Para la caracterización hidrográfica de la columna de agua, los registros de temperatura, salinidad, densidad y clorofila-a fueron corregidos a través de procedimientos estándares de acuerdo a CENDHOC CHILE, con el programa Data processing (SEABIRD) y promediados cada un metro. A partir de estos, se realizaron secciones transversales con cada una de las variables utilizando el programa Ocean Data View (Schlitzer 2008). Debido al discreto esfuerzo muestreal y la mayor distancia geográfica entre las estaciones E8 y E9, los valores de los STS $y$ nutrientes no fueron extrapolados entre estas dos estaciones.

Análisis estadístico

Para determinar la combinación de variables ambientales que expliquen mejor la variación de la concentración de Chl-a a lo largo de seno Gallegos se aplicó la rutina BEST del programa PRIMER v6.0 (Clarke \& Gorley 2006). La rutina BEST realiza una exhaustiva búsqueda entre todas las posibles combinaciones de variables ambientales seleccionando aquellas combinaciones que mejor se correlacionen con la matriz de similitud de la variable biótica. Las matrices de similitud de Chl-a y de las variables ambientales se calcularon usando distancias euclidianas, previa transformación con raíz cuadrada. Posteriormente, la correlación se realizo usando Spearman ( $\rho$ ) y a través de la prueba de permutación, integrado en la rutina BEST, se calculo con nivel de significancia al 95\% (Clarke \& Gorley 2006).

\section{RESULTADOS}

La columna de agua del seno Gallegos presentó un rango térmico desde 3,74 a $6,55^{\circ} \mathrm{C}$, el rango de salinidad fue de 28,39 - 30,14 psu y la concentración de clorofila a varió entre 0,24 - 2,69 $\mathrm{mg} \mathrm{m}^{-3}$. Mientras que la concentración de los STS variaron entre los 0,12 y $14,0 \mathrm{mg} \mathrm{L}^{-1}$. En cuanto a los nutrientes, la concentración de silicatos varió entre 2,58 - 9,57 $\mu \mathrm{M}$, mientras la concentración de fosfatos varió entre $0,01-8,02 \mu \mathrm{M}$. La concentración de nitratos y nitritos presentaron una variación de $0,02-30,34 \mu \mathrm{M}$ y $0,01-2,99 \mu \mathrm{M}$, respectivamente. La profundidad mínima de muestreo fue de 11 metros y la mayor fue a los 62 metros de profundidad (Tabla 1). 


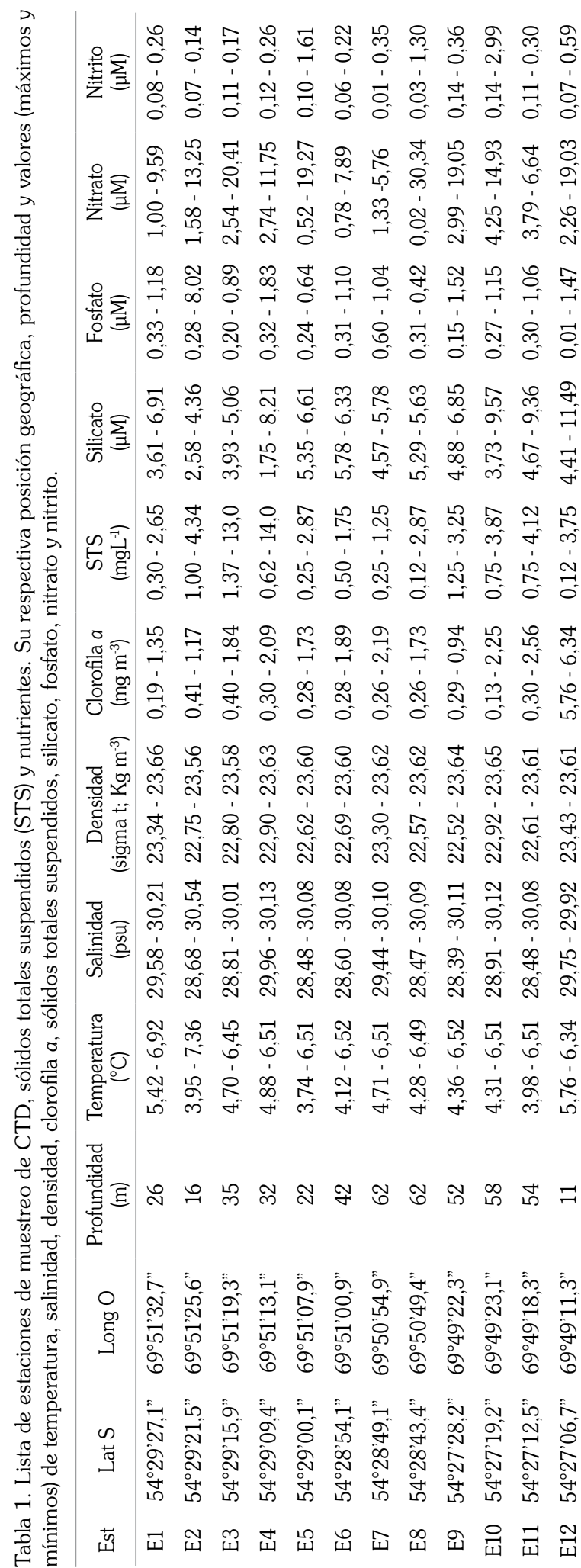

Características físico-químicas de la columna de agua.

La columna de agua en seno Gallegos se dividió en dos capas, una capa superficial hasta los $15 \mathrm{~m}$ aproximadamente, la cual presentó una mayor variabilidad térmica, registrando en los primeros 5 $\mathrm{m}$ temperaturas con valores entre los 3,74 y $5,5^{\circ} \mathrm{C}$; mientras que la capa profunda fue más estable y casi homotermal con temperaturas desde 6,3 hasta $6,56^{\circ} \mathrm{C}$. En las estaciones cercanas al glaciar (E1, E2, E3, E4, E5, E6, E7, E8) la capa superficial presentó un espesor de $15 \mathrm{~m}$ con temperaturas entre 3,74 y $6,51^{\circ} \mathrm{C}$, esta capa disminuyó su espesor a $10 \mathrm{~m}$ en las estaciones menos expuestas (E9, E10, $\mathrm{E} 11, \mathrm{E} 12)$, las que presentaron un rango de 3,98 a $6,30^{\circ} \mathrm{C}$ (Fig. 2A).

Similar a lo observado con la temperatura, la salinidad presenta mayor variabilidad en los primeros metros, con valores entre 28,39 y 29,95 psu; por tanto una tenue haloclina se distinguió en los primeros $5 \mathrm{~m}$, la cual se profundizó hasta los $10 \mathrm{~m}$ hacia la boca del seno. En contraste, la capa profunda fue prácticamente homogénea con valores superiores a 29,95 psu y con un valor máximo de 30,14 psu (Fig. 2B).

Los valores de densidad superficial a lo largo del seno mostraron un rango entre 22,52 a 23,67 kg $\mathrm{m}^{-3}$. Bajo esta capa, se observó una leve picnoclina entre los 0 y $5 \mathrm{~m}$ en las estaciones E8 y E9 con valores entre 22,52 y $23,3 \mathrm{~kg} \mathrm{~m}^{-3}$, mientras que el menor gradiente se presentó en las estaciones E1, $\mathrm{E} 2, \mathrm{E} 3, \mathrm{E} 4, \mathrm{E} 5$ y E6 entre los 0 y $5 \mathrm{~m}$ con valores desde 22,9 hasta $23,3 \mathrm{~kg} \mathrm{~m}^{-3}$ (Fig. 2C).

Patrón de distribución de las concentraciones de Chl- $a$, STS y nutrientes.

Las mayores concentraciones de Chl-a se registraron en los primeros $5 \mathrm{~m}$ de la columna de agua, con un valor máximo de $2,69 \mathrm{mg} \mathrm{m}^{-3}$ (E9), bajo los $10 \mathrm{~m}$ las concentraciones fueron inferiores a 1 $\mathrm{mg} \mathrm{m}^{-3}$. Se observó un aumento en la concentración de Chl-a desde la cabeza (en E6, E7, E8; 0,57 mg $\mathrm{m}^{-3}$ ) a la boca del seno con un valor máximo de 2,69 $\mathrm{mg} \mathrm{m}^{-3}$ (E9, E10, E11, E12) (Fig. 2D).

Los mayores contenidos de STS se presentaron en el área adyacente al glaciar (E1, E2, E3, E4)

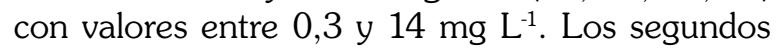




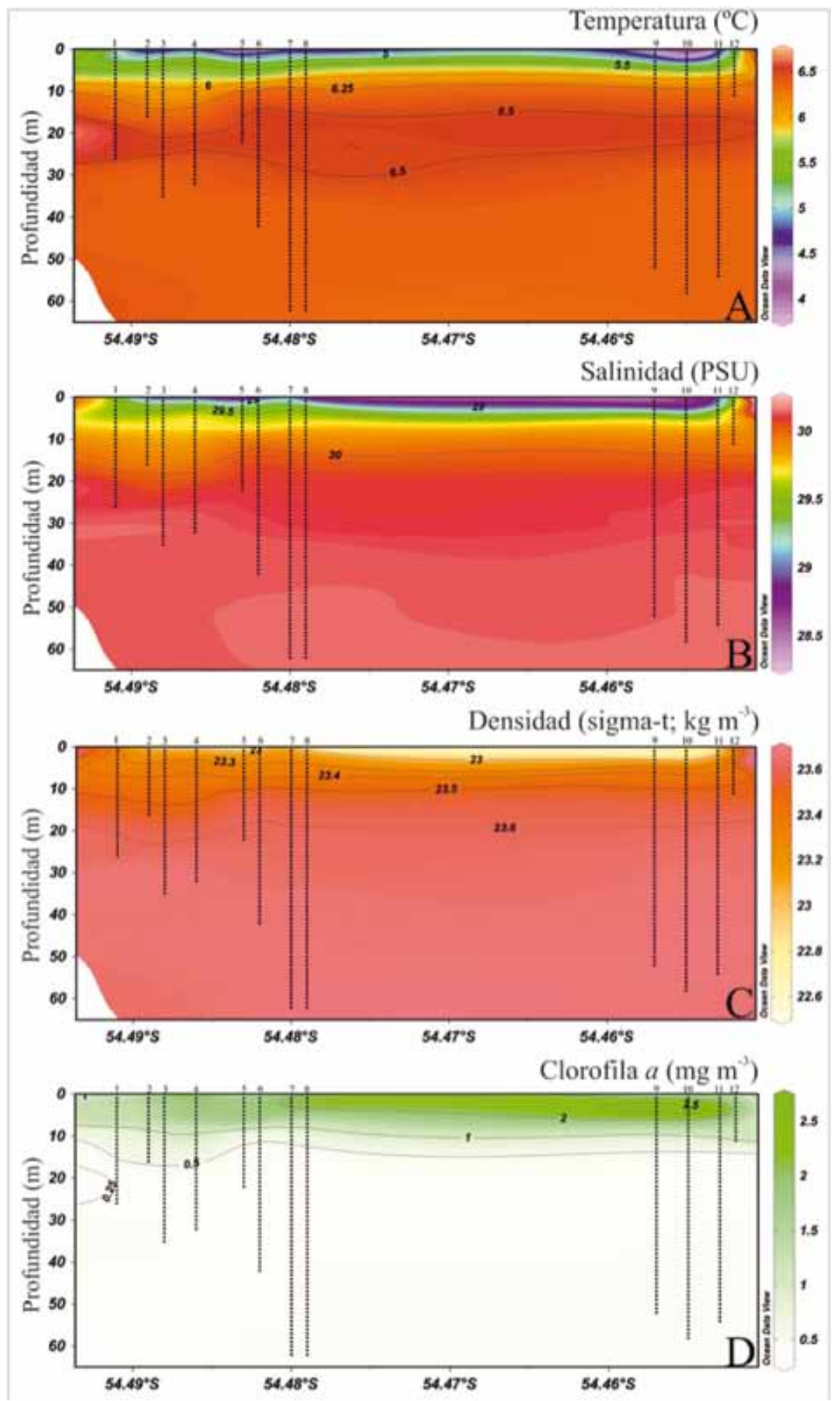

Fig. 2. Distribución vertical de temperatura $\left({ }^{\circ} \mathrm{C}\right.$ ), salinidad (psu), densidad (sigma-t; $\left.\mathrm{kg} \mathrm{m}^{-3}\right)$ y clorofila $a\left(\mathrm{mg} \mathrm{m}^{-3}\right)$ desde la cabeza (E1) a la boca (E12) del seno Gallegos bajo condiciones de invierno. 


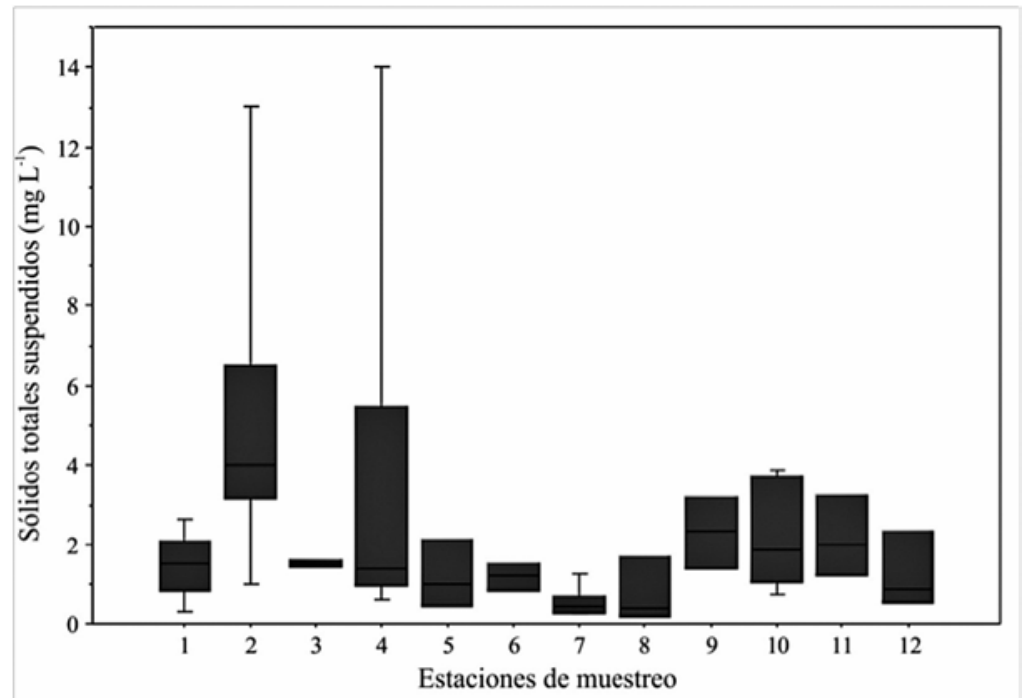

Fig. 3. Contenido de los sólidos totales suspendidos (STS) desde la cabeza (E1) a la boca (E12) del seno Gallegos bajo condiciones de invierno. Las líneas horizontales representan los cuartiles de distribución (10, 25, 50,75 y 90) de la variable $(\mathrm{N}=60)$.

contenidos más altas se obtuvieron en el área menos expuesta con valores entre 0,125 a 4,125 $\mathrm{mg} \mathrm{L}^{-1}$ (E9, E10, E11, E12), mientras que los contenidos más bajas se encontraron entre ambas áreas, con valores entre 0,125 a 2,87 $\mathrm{mg} \mathrm{L}^{-1}$ (E5, E6, E7, E8) (Fig. 3).

Los valores de silicatos (Fig. 4A) presentaron baja variación vertical a lo largo del seno, donde las estaciones de menor exposición al glaciar (i.e., boca del seno) presentaron un valor mínimo de $4,18 \mu \mathrm{M}$ y máximo de $8,69 \mu \mathrm{M}$ (superficie y $10 \mathrm{~m}$, respectivamente), mientras que la variación vertical presente en la estación con exposición intermedia (E5, E6, E7 y E8) fue más baja. Por otro lado, las estaciones con mayor exposición, muestran mayor concentración en superficie $(5,79 \mu \mathrm{M})$ y el menor valor a los $10 \mathrm{~m}(3,81 \mu \mathrm{M})$.

Las concentraciones de fosfato presentaron una alta variación vertical con un rango de 0,22 a 2,85 $\mu \mathrm{M}$ a lo largo del seno. En la zona más expuesta se observó la mayor concentración en la superficie, lo que disminuye a valores menores de $1 \mu \mathrm{M}$ a lo largo del seno, sin embargo, hay un leve aumento de la concentración entre los 20 y 30 m de profundidad de la de las estaciones con menor exposición (Fig. 4B)

La concentración de nitratos (Fig. 4C) varió entre 2,82 y $10,34 \mu \mathrm{M}$ y presentó una mayor variación vertical en la estación de influencia intermedia (E5 a E8), donde se observaron las mayores concentraciones, bajo los $15 \mathrm{~m}$. Por su parte, las estaciones correspondientes a las de mayor y menor exposición al glaciar fueron más homogéneas, con valores que fluctuaron entre 5,09 a 9,11 $\mu \mathrm{M}$, respectivamente. Mientras que, los nitritos (Fig. 4D) mostraron la mayor concentración a los 5 $\mathrm{m}(1,07 \mu \mathrm{M})$ en la estación de menor exposición al glaciar (boca del seno), y el menor valor (a los $5 \mathrm{~m}$ ) en las estaciones de exposición intermedia $(0,09 \mu \mathrm{M})$. En general, la concentración vertical de nitrito fue mayor en las estaciones cercanas a la boca del seno.

Relación entre la concentración de Chl-a, con los STS y nutrientes.

A través de la rutina BEST se logró determinar la correlación de las variables ambientales con la distribución vertical y horizontal de Chl-a en seno Gallegos. La tabla 2 muestra las 10 mejores combinaciones, donde las variables profundidad junto con STS y densidad muestran una correlación significativa $(\rho=0,477 ; p=0,01)$ con la Chl- $a$. En el caso de las variables químicas, las combinaciones que presentaron los mejores valores de correlación fueron los silicatos y fosfatos $(\rho=0,472 ; p=0,01$ y $\rho=0,463 ; p=0,01$, respectivamente), mientras que las combinaciones con menor valor $\rho$ fueron las que agruparon a los nitratos $(\rho=0,397 ; p=0,01)$. 

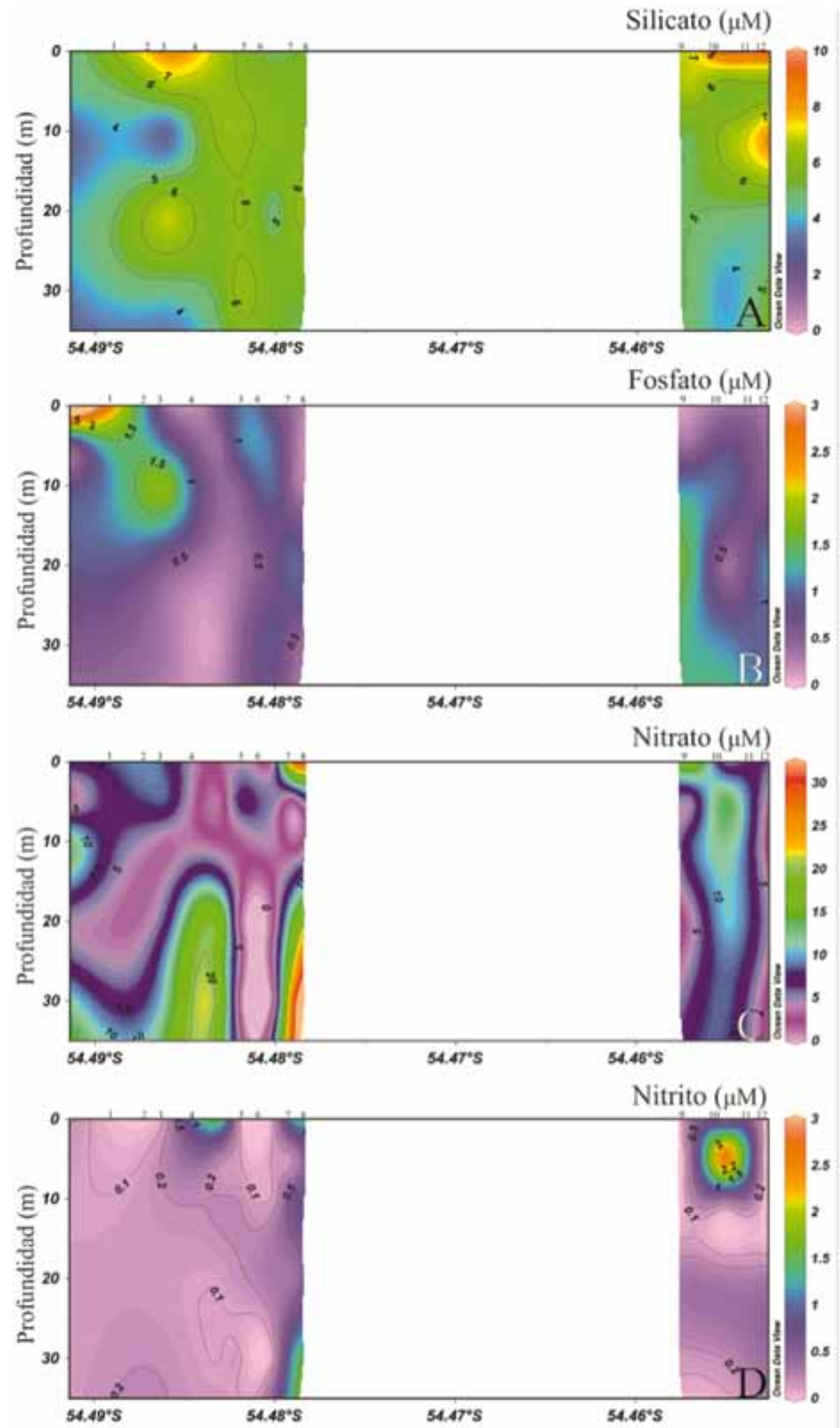

Fig. 4. Distribución vertical de silicato $(\mu \mathrm{M})$, fosfato $(\mu \mathrm{M})$, nitrato $(\mu \mathrm{M})$ y nitrito $(\mu \mathrm{M})$ desde la cabeza (E1) a la boca (E12) del seno Gallegos bajo condiciones de invierno $(\mathrm{N}=60)$. 
Tabla 2. Valores de correlación de Spearmen (rho) entre la Chl-a y las mejores combinaciones de variables oceanográficas obtenidas a través de la rutina BEST. (* $p=0,01)$.

\begin{tabular}{ccc}
\hline Tipo de variable & Variables seleccionadas por rutina BEST & Correlación \\
\hline Chl- $a$ & Profundidad, STS, Densidad & $0,477^{*}$ \\
Chl- $a$ & Profundidad, STS & $0,477^{*}$ \\
Chl- $a$ & Profundidad, Silicato, STS & $0,472^{*}$ \\
Chl- $a$ & Profundidad, Silicato, STS, Densidad & $0,471^{*}$ \\
Chl- $a$ & Profundidad, Fosfato, STS & $0,463^{*}$ \\
Chl- $a$ & Profundidad, Fosfato, STS, Densidad & $0,463^{*}$ \\
Chl- $a$ & Profundidad, Fosfato, Silicato, STS & $0,456^{*}$ \\
Chl- $a$ & Profundidad, Fosfato, Silicato, STS, Densidad & $0,456^{*}$ \\
Chl- $a$ & Profundidad, Nitrato, STS & $0,397^{*}$ \\
Chl- $a$ & Profundidad, Nitrato, STS, Densidad & $0,397^{*}$ \\
\hline
\end{tabular}

\section{DISCUSIÓN}

La presencia del Campo de Hielo Cordillera Darwin juega un rol importante en la dinámica oceanográfica de las aguas adyacentes a este, ya que aporta gran cantidad de agua de deshielo hacia el canal Beagle, seno Agostini y seno Almirantazgo, esto sumado a la pluviosidad y escurrimientos típicos de la zona, provocan una alteración en las características oceanográficas de los fiordos interiores (Valdenegro \& Silva 2003).

En la capa superficial de seno Gallegos, la distribución horizontal de temperatura y salinidad presenta características típicas de una zona estuarina bajo condiciones de invierno, donde la influencia de la densa capa de hielo marino formada en la zona aledaña al glaciar y a la escarcha en el resto del seno, provocan una diminución de la temperatura y aumento de la salinidad en la capa superficial (Silva \& Calvete 2002; Avaria et al. 2004; Calvete \& Sobarzo 2011). Sin embargo, el leve aumento de temperatura y la disminución de la salinidad en la boca del seno (estaciones E1, E7 y E12) se deben a factores locales asociados a la presencia de pequeños cuerpos de agua dulce dentro del seno y la presencia de otro glaciar en la bahía adyacente, los que aportarían agua dulce más fría.

A lo largo de todo el seno Gallegos se observó una inversión térmica, lo que de acuerdo a Sievers (2006), es atribuible a la menor radiación solar de las latitudes intermedias. Este patrón también ha sido descrito para otras localidades como el canal Pulluche-Chacabuco (Silva \& Guerra 2008) y seno Aysén (Sievers \& Prado 1994) en la temporada de invierno. Sin embargo, estos presentaron una menor variabilidad de la temperatura $(9,3$ - 10,2 ${ }^{\circ} \mathrm{C}$; Silva \& Guerra 2008) que seno Gallegos (3,74 $\left.-6,56^{\circ} \mathrm{C}\right)$. Se espera que con el aumento de la irradiancia en las temporadas de primavera y verano, y por ende un mayor calentamiento superficial de la columna de agua, la condición de inversión térmica se modifique, dado que la temperatura de la capa superficial será más cálida que la capa profunda (Sievers, 2006). Los valores superficiales de temperatura reportados para este trabajo son similares a los descritos por Andrade (1991) en invierno para bahía Brookes con valores entre 3 - 5 ${ }^{\circ} \mathrm{C}$, sin embargo, al comparar los valores de seno Gallegos con datos obtenidos en bahías adyacentes (Bahía Parry), en temporada de primavera, son al menos $2{ }^{\circ} \mathrm{C}$ más bajos (Valdenegro \& Silva 2003) con valores superiores a $5,5^{\circ} \mathrm{C}$.

Los valores de salinidad muestran una estructura de dos capas separadas por una leve haloclina, donde la influencia de la densa capa de hielo en las estaciones más expuestas (i.e., cabeza del seno), produce un aumento de la salinidad superficial de estas estaciones, aunque sigue siendo menor que la capa profunda. En tanto, la disminución de la salinidad en las estaciones cercanas a la boca del seno está relacionada a la presencia de pequeños cuerpos de agua, los que aportarían agua dulce. Patrones similares a este han sido descritos para la zona de fiordos chilenos (Pickard 1971), para seno Ballena (Valle-Levinson et al. 2006), para fiordos de Alaska (Matthews 1981) y fiordos de Noruega (Stigebrandt 1990). Por otra parte, en la época de estudio, asociada a la formación del hielo marino, se produce un aumento general de la salinidad lo que causa un posible ingreso de agua más salina en la 
capa profunda desde bahía Brookes, cuyo valor de salinidad entre los meses de septiembre y octubre es 31\%o (Andrade 1991).

Las pequeñas variaciones de temperatura y salinidad en la capa superficial a lo largo del seno, originan una densidad superficial homogénea, con valores desde 22,52 a $23 \mathrm{~kg} \mathrm{~m}^{-3}$ entre las estaciones $\mathrm{E} 1$ y E12, mientras que, verticalmente las mayores oscilaciones de temperatura pueden influir en las variaciones de densidad en los primeros $10 \mathrm{~m}$, con valores entre 22,5 - 23,5 $\mathrm{kg} \mathrm{m}^{-3}$. Este patrón es distinto al observado, en la temporada de invierno, para seno Aysén, donde los valores aumentaron desde la cabeza a la boca del fiordo, con valores desde 5 a $20 \mathrm{~kg} \mathrm{~m}^{-3}$ (Sievers \& Prado 1994) y para el transecto desde el Golfo Elefantes a la boca del Guafo (Región de Aysén), donde se observa un claro aumento hacia la zona oceánica, las que presentan valores superiores a $23 \mathrm{~kg} \mathrm{~m}^{-3}$ (González et al. 2011). Esta diferencia puede estar asociada a que tanto la extensión como la profundidad de estos senos son mayor a la encontrada de seno Gallegos.

Las concentraciones de Chl-a en seno Gallegos, con un rango de 0,24 - 2,69 $\mathrm{mg} \mathrm{m}^{-3}$, son más altas que las reportadas por Czypionka et al. (2011) para el fiordo Aysén con una concentración máxima de $1,48 \mathrm{mg} \mathrm{m}^{-3}$ en superficie $(0 \mathrm{~m})$ y por Pizarro et al. (2005) para la zona norte de los fiordos y canales $\left(<1 \mathrm{mg} \mathrm{m}^{-3}\right)$, quienes lo relacionan a la disminución de la irradiancia medida durante la temporada de invierno. Sin embargo, también son más altos que los registrados para el estrecho de Magallanes en primavera donde se registró un promedio de $0,32 \mathrm{mg} \mathrm{m}^{-3}$ (Saggiomo et al. 2011) y más bajos que los reportados por Torres et al. (2011) para seno Ballena, con un valor máximo de $8 \mathrm{mg} \mathrm{m}^{-3}$ (primavera-verano).

Por otro lado, la variabilidad que presenta la distribución de la Chl-a, a lo largo de seno Gallegos, se podría explicar por la gran cantidad de material en suspensión en las estaciones aledañas al glaciar, lo que es característico de zonas periglaciares (Pickard 1971). Este material se genera por procesos de erosión propios de estas áreas, lo que provoca una reducción en la penetración de la luz, limitando la producción local de Chl-a (Pizarro et al. 2005). Algunos estudios sugieren que los mecanismos propuestos para explicar los patrones de distribución del fitoplancton depen- den de (i) la secuencia cronológica del muestreo realizado en una microcuenca determinada, (ii) las secuencia cronológica de la fase de marea en la microcuenca, (iii) el emplazamiento físico que evidencie el intercambio de las aguas de la microcuenca con las del estrecho de Magallanes y (iv) los antecedentes batimétricos que permitan detectar la influencia de la profundidad sobre el proceso de mezcla (e.g., Ramírez 2005). En este sentido, basados en los resultados de la correlación entre la clorofila a y las variables ambientales, es posible señalar que tanto la profundidad de la columna de agua como los STS son los factores que podrían estar influyendo mayormente en la distribución de esta, dado que aparecen en todas las combinaciones con mejor correlación (Tabla 2). Sin embrago, no explican completamente la distribución de la misma, debido a que la densidad (estabilidad de la columna de agua) es el segundo parámetro que aparece más veces con buena correlación en estas combinaciones, dado que contribuye a mantener las concentraciones de Chl-a en la capa superficial, disminuyendo la sedimentación celular (Ramírez 2005). Otro parámetro importante que influye en la concentración y distribución de la clorofila a en la columna de agua son los nutrientes, donde las mejores combinaciones fueron las de silicatos y fosfatos en comparaciones con la de nitratos los cuales tuvieron una baja correlación (ver Tabla 2).

La distribución de los nutrientes muestra que las mayores concentraciones de silicatos se observaron en la capa superficial de las estaciones menos expuestas (i.e., boca del seno), lo que puede estar relacionado con la influencia de las aguas de la bahía adyacente provenientes de los escurrimientos costeros y el aporte fluvial. Además, la mayor concentración de silicatos en la boca del seno y su disminución hacia la estación E9 puede estar relacionada con la mayor concentración de Chl-a, dado que existe un consumo por parte del fitoplancton (Silva \& Calvete 2002), lo que además está asociado a una mayor concentración de la componente nitrogenada (nitrato y nitrito) en estas estaciones. En este sentido, al observar los valores de correlación en la combinación de los parámetros profundidad, STS y los nutrientes, los silicatos presentaron la mejor correlación $(\rho=0,472)$, lo que sugiere la presencia de fitoplancton con estructuras de sílice (diatomeas) al interior del seno, tal como se ha sido descrito por 
Frangópulos et al. (2007)3 para otras áreas (e.g., seno Ballena). Por otro lado, los fosfatos presentan las mayores concentraciones superficiales en las estaciones más expuestas, bajo esta capa se produce un mínimo subsuperficial producto del ingreso de masas de agua con menor contenido de fosfato. La distribución vertical, presentó un máximo en superficie disminuyendo con la profundidad, sin embargo, en el centro del seno se observa un aumento en los $30 \mathrm{~m}$, lo que se puede estar asociado a una remineralización de la materia orgánica en el sedimento (Antezana 1999). Las estaciones aledañas al glaciar presentaron bajas concentraciones, debido al pobre aporte del escurrimiento desde el glaciar asociado a la formación de hielo durante esta temporada.

En general, durante la temporada de invierno, se puede señalar que a pesar de existir una combinación de variables que influyen sobre la concentración de Chl-a, su distribución en seno Gallegos estaría dominada por la combinación de los parámetros profundidad y STS con una alta correlación ( $\rho=$ $0,477)$ junto con la densidad $(\rho=0,477)$, dado que la estratificación es una barrera que mantiene al fitoplancton en la capa superficial, mientras que los STS limitan la penetración de la luz (Ramirez 2005). Por otra parte, la combinación de silicatos, STS y profundidad presentan la tercera mejor combinación ( $\rho=0,472)$, lo que está asociado al sílice que aporta el material terrígeno proveniente de la cuenca de los glaciares y al uso de este nutriente por el fitoplancton (Frangópulos et al. 2007).

\section{CONCLUSIONES}

La columna de agua en seno Gallegos presenta una estructura vertical de dos capas, donde la capa superficial está influenciada por una masa de hielo-marino en las zonas más expuestas al glaciar y escarcha en las zonas menos expuestas.

La estratificación de la columna de agua en combinación con los STS y la profundidad presentes durante el periodo de estudio, contribuye a mantener

3 Frangópulos, M., Blanco, J., Hamamé, M., Rosales, S., Torres, R. \& Valle-Levinson, A. 2007. Análisis y diagnóstico de las principales características oceanográficas del área marina costera protegida Francisco Coloane, Informe Final Proyecto GEF-PNUD "Conservación de la Biodiversidad de Importancia Mundial a lo Largo de la Costa Chilena”. 122. Manuscrito no publicado. altas las concentraciones de Chl-a en la capa fótica (capa superficial) del seno Gallegos.

Por otro lado, las combinaciones que incluyen a los silicatos explican significativamente la distribución de las concentraciones de Chl-a. Mientras que, en menor grado, las combinación que incluyen a los fosfatos y por último los nitratos.

\section{AGRADECIMIENTOS}

Se agradece enormemente al capitán Alejandro González y a la tripulación de la L/M Cabo Tamar por la cooperación prestada durante los muestreos. A la Fundación CEQUA (Centro de Estudio del Cuaternario Fuego Patagónico y Antártica) por facilitar los equipos necesarios para esta investigación. Esta investigación fue financiada por el proyecto FONDECYT iniciación 11090208 (A. Montiel). Los autores deseamos agradecer a los árbitros anónimos por sus valiosas sugerencias para mejorar este artículo.

\section{LITERATURA CITADA}

Andrade, S. 1991. Geomorfología costera y antecedentes oceanográficos físicos de la región de Magallanes, Chile (48 $\left.-56^{\circ} \mathrm{S}\right)$. Anales Instituto Patagonia 20 (1):136-151

Antezana, T. 1999. Hydrographic features of Magellan and Fuegian inland passages and adjacent Subantarctic waters. Scientia Marina 63 (1):23-34

Avaria, S., C. Cáceres \& P. Muñoz 2004. Distribución del microfitoplancton marino entre el Golfo Corcovado y el Estero Elefantes en la primavera de 1998 y en el verano de 1999 (Crucero CIMAR-4 fiordos). Revista Ciencia y Tecnología del Mar 27 (1):17-47

Calvete, C. \& M. Sobarzo 2011. Quantification of the surface brackish water layer and frontal zones in southern Chilean fjords between Boca del Guafo (43 $30^{\prime} \mathrm{S}$ ) and Estero Elefantes (46 30'S). Continental Shelf Research 31:162-171

Castro, P. \& M. Huber 2003. Chemical and Physical Features of Seawater and the World Ocean. En: Marine biology. Fourth edition. The McGraw- Hill Companies:48-71

Chuecas, L. \& R. Ahumada 1980. Contribución al conocimiento de las condiciones hidrográficas de los fiordos de la región magallánica- 
Chile. Boletim do Instituto Oceanografico 29 (2):95-100

Clarke, K. \& N. Gorley 2006. PRIMER v6: User Manual/Tutorial. PRIMER-E: Plymouth. 182

Czypionka, T., C. Vargas, N. Silva, G. Daneri, H. González \& J. Iriarte 2011. Importance of mixotrophic nanoplankton in Aysen Fjord (Southern Chile) during austral winter. Continental Shelf Research 31:216-224

Eaton, A., L. Clesceri, E. Rice \& A. Greenberg 2005. Standard methods for the examination of water \& wastewater. $21^{\text {st }}$ edition, centennial edition.1325pp

González, H., F. Castro, G. Daneri, J. Iriarte, N. Silva, C. Vargas, R. Giesecke \& N. Sánchez 2011. Seasonal plankton variability in Chilean Patagonia fjords: Carbon flow through the pelagic food web of Aysen Fjord and plankton dynamics in the Moraleda Channel basin. Continental Shelf Research 31:225-243

Guzmán, D. \& N. Silva 2002. Caracterización física y química y masas de agua en los canales australes de Chile entre Boca del Guafo y Golfo Elefantes (Crucero CIMAR-4 fiordos). Revista Ciencia y Tecnología del Mar 25(2): 45-76

Iriarte, J., H. González, K. Liu, C. Rivas \& C. Valenzuela 2007. Spatial and temporal variability of chlorophyll and primary productivity in surface waters of southern Chile (41.5-43ㅇ). Estuarine, Coastal and Shelf Science 74:471-480

Kilian, R., Baeza O., Steinke T., Arevalo M., Rios C. \& Schneider, C. 2007. Late Pleistocene to Holocene marine transgression and thermohaline control on sediment transport in the western Magellanes fjord system of Chile $\left(53^{\circ} \mathrm{S}\right)$. Quaternary International 161:90-107

McLusky, D. \& M. Elliot 2004. The estuarine ecosystem; ecology, threats and management, third edition. Published in the United States by Oxford University Press Inc., New York. 111-223pp

Matsuura, H. \& G. Cannon 1997. Wind effects on sub-tidal currents in Budget Sound. Journal Oceanography 53:55-66

Matthews, J. 1981. The Seasonal Circulation of the Glacier Bay, Alaska Fjord System. Estuarine, Coastal and Shelf Science 12:679-700

Murray, J. 1895. Report on the scientific results of the Voyage of H.M.S Challenger during the years 1872-76. A summary of the scientific results. Pt 2.797-1608pp

Pantoja, S., J. Iriarte \& G. Daneri 2011. Oceanography of the Chilean Patagonia. Continental Shelf Research 31:149-153

Parsons, T., Y. Maita \& C. Lalli 1994. A manual of chemical and biological methods for seawater analysis. Pergamon Press, Oxford. 173pp

Pickard, G. 1971. Some physical oceanographic features of inlet of Chile. Journal of Fisheries Research Board of Canada 28:1077-1106

Pizarro, G., R. Astoreca, V. Montecino, M. Paredes, G. Alarcón, P. Uribe \& L. Guzmán 2005. Patrones espaciales de la abundancia de la clorofila, su relación con la productividad primaria y la estructura de tamaño del fitoplancton en julio y noviembre de 2001 en la región de Aysén $\left(43^{\circ}-46^{\circ} \mathrm{S}\right)$. Revista Ciencia y Tecnología del Mar 28 (2):27-42

Ramírez, B. 2005. Distribución vertical de clorofila en los fiordos australes ubicados entre el Estrecho de Magallanes y el Cabo de Hornos (Chile). Revista Ciencia y Tecnología del Mar 28 (2):43-61

Rignot, E., A. Rivera \& G. Casassa 2003. Contribution of the Patagonia Icefields of South America to Sea Level Rise. Science 302:434-437

Saggiomo, V., I. Santarpia, M. Saggiomo, F. Margiotta \& O. Mangoni 2011. Primary production processes and photosynthetic performance of a unique periantarctic ecosystem: the Strait of Magellan. Polar Biology 34:1255-1267

Schlitzer, R. 2008. Ocean Data View User's Guide v3.4.0. Alfred Wegener Institute. 96pp

Sievers, H. 2006. Temperatura y salinidad en canales y fiordos australes. Avances en el conocimiento oceanográfico de las aguas interiores chilenas, Puerto Montt a cabo de Hornos. N. Silva \& S. Palma (eds). Comité Oceanográfico Nacional-Pontificia Universidad Católica de Valparaíso, Valparaíso:31$36 \mathrm{pp}$

Sievers, H., C. Calvete \& N. Silva 2002. Distribución de características físicas, masas de agua y circulación general para algunos canales australes entre el Golfo de Penas y el Estrecho de Magallanes (Crucero CIMAR-2 fiordos), Chile. Revista Ciencia y Tecnología del Mar 25 (2):1-43 
Sievers, H. \& R. Prado 1994. Contraste de las características oceanográficas del Seno Aysén, Chile, entre invierno y verano (lat. $45^{\circ}$ 20'S). Revista Biología Marina, Valparaíso 29 (2):167-209

Silva, N. \& D. Guerra 2008. Distribución de temperatura, salinidad, oxígeno disuelto y nutrientes en el canal Pulluche-Chacabuco, Chile. (Crucero CIMAR 9 fiordos). Revista Ciencia y Tecnología del Mar 31 (2):29-43

Silva, N \& S. Palma 2006. El Programa CIMAR en los canales y fiordos australes. Avances en el conocimiento oceanográfico de las aguas interiores chilenas, Puerto Montt a Cabo de Hornos. N. Silva \& S. Palma (eds.). Comité Oceanográfico Nacional-Pontificia Universidad Católica de Valparaíso, Valparaíso:11-15pp

Silva, N. \& C. Calvete 2002. Características oceanográficas físicas y químicas de canales australes chilenos entre el Golfo de Penas y el Estrecho de Magallanes (Crucero CIMAR-2 fiordos). Revista Ciencia y Tecnología del Mar 25 (1):23-88

Silva, N., V. De Vidts \& J. Sepúlveda 2001. Materia orgánica, Cy N, su distribución y estequiometria, en sedimentos superficiales de la región central de los fiordos y canales australes de Chile (Crucero CIMAR-2 fiordos). Revista Ciencia y Tecnología del Mar 24:23-40

Sobarzo, M. 2009. La región de los fiordos de la zona sur de Chile: Aspectos oceanográficos.
En Vreni Häussermann \& Günter Försterra (eds) Fauna bentónica de la Patagonia Chilena:53-60pp

Stigebrandt, A. 1990. On the response of the horizontal mean vertical density distribution in a fjord to low-frequency density fluctuations in the coastal water. Tellus. 42a:605-614

Strickland, J. \& T. Parsons 1972. A practical handbook of seawater analysis. $2^{\text {nd }} e d$. Bulletin Fisheries Research Broad of Canada. $167 \mathrm{pp}$

Strickland, J. \& T. Parsons 1968. A Practical Handbook of Seawater Analysis. Bulletin Fisheries Research Broad of Canada. 167pp

Torres, R., M. Frangópulos, M. Hamamé, V. Montecino, C. Maureira, G. Pizarro, B. Reid, A. Valle-Levinson \& J. Blanco 2011. Nitrate to silicate ratio variability and the composition of micro-phytoplankton blooms in the inner-fjord of Seno Ballena (Strait of Magellan, $54^{\circ} \mathrm{S}$ ). Continental Shelf Research 31:244-253

Valdenegro, A. \& N. Silva 2003. Caracterización oceanográfica física y química de la zona de canales y fiordos australes de Chile entre el Estrecho de Magallanes y Cabo de Hornos (CIMAR-3 fiordos). Revista Ciencia y Tecnología del Mar 26 (2):19-60

Valle-Levinson, A., J. Blanco \& M. Frangópulos 2006. Hydrography and frontogenesis in a glacial fjord off the Strait of Magellan. Ocean Dynamics 56:217-227 
